\title{
Tidal Tails of the Nearest Open Clusters
}

\author{
Yaroslav Chumak $^{1}$ and Alexey Rastorguev ${ }^{2}$ \\ ${ }^{1}$ Sternberg Astronomical Institute, Universitetskiy prospect 13, Moscow,119992, Russia \\ email:chyo@mail.ru \\ ${ }^{2}$ Department of Physics, Moscow State University, GSP-2, Leninskiye Gory, Moscow, 119992, \\ Russia \\ email:rastor@sai.msu.ru
}

\begin{abstract}
We show that an extended population of stars escaping an evolved cluster and moving along its galactic orbit forms at the final phases of its dynamical evolution. Here we present some results of the numerical simulations for nearest open clusters: Hyades, Pleiades, Praesepe, Alpha Persei, Coma, IC 2391, and IC 2602. We calculated the models of the stellar tails for nearest open clusters and estimated some parameters: sizes, densities, locations relative to the solar neighborhood. Stars of the nearest tails can be observed as moving clusters.
\end{abstract}

Keywords. open clusters and associations: general, methods: n-body simulations

In our simulations (Chumak, Rastorguev, \& Aarseth 2005), (Chumak \& Rastorguev 2006a) it was shown that stars escaped from a cluster at different times, move very close to cluster's orbit. Their relative velocities are small but relative distances along the cluster orbit change from parsecs, to more than $1.5 \mathrm{kpc}$. As a consequence, escaped stars form a stretched stellar tail, which can exist for a fairly long time even after full disintegration of the open cluster as its relic. We performed simulations of the dynamical evolution of seven nearest clusters in the tidal field of the Galaxy (Chumak \& Rastorguev 2006b). Our computations of the dynamical evolution have been based on the Aarseth's code NBODY6 (Aarseth 2003), with known cluster age estimates and real Galactic orbits. Initial conditions have been chosen in such a way that current parameters of simulated clusters corresponded to their observed parameters (Nordstrom et al. 2004).

Fig. 1 (left panel) shows the position of stellar tail relative to the cluster and its orbit for typical cluster with an age $1233 \mathrm{Myr}$. The right panel shows the close neighborhood of the cluster, $\pm 200 \mathrm{pc}$.

The results can be briefly summarized as follows:

1. The length of the cluster tails along the Galactic orbit, its thickness (along the $Z$ axis), and its width (along the $X$ axis) for a typical cluster (with $N=1500$, initial virial radius $R V=3.5 \mathrm{pc}$, an age of $800 \mathrm{Myr}$ ) can reach $L Y=900 \mathrm{pc}, L Z=20 \mathrm{pc}$, and $L X=100 \mathrm{pc}$, respectively.

2. The stars in the tail may be considered as moving clusters, since their mean velocity vector is close to the velocity vector of the parent cluster and their internal velocity dispersion is relatively low $\left(\sim 1 \mathrm{~km} \mathrm{~s}^{-1}\right)$.

3. Hyades. The Hyades cluster lies inside the circumsolar sphere with the radius of $100 \mathrm{pc}$, and the cluster tail crosses this sphere and extends far beyond in both directions (see Fig. 2). The approximate length of the tail along the $Y$ axis that falls within the circumsolar sphere is $\sim 160$ pc. This sphere contains approximately 150 tail stars.

4. Coma. The tail of Coma cluster also crosses the circumsolar sphere. The approximate length of the tail along the $\mathrm{Y}$ axis that falls within the circumsolar sphere is $\sim 80 \mathrm{pc}$. The sphere contains about 80 tail stars. 

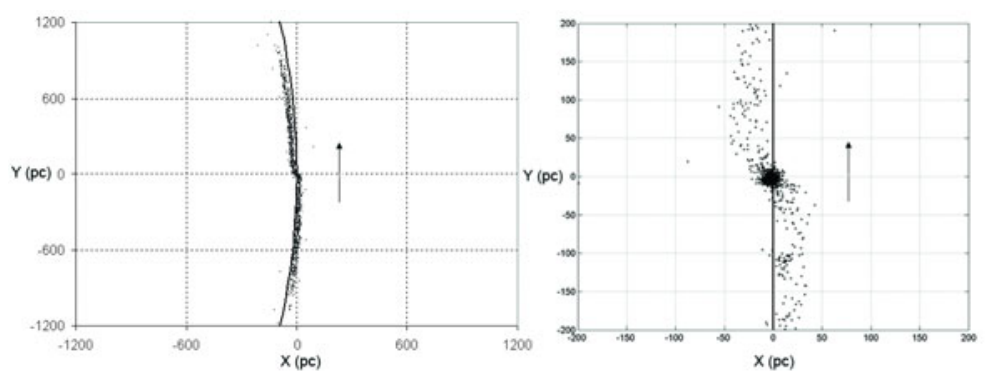

Figure 1. Typical stellar tail for open clusters.

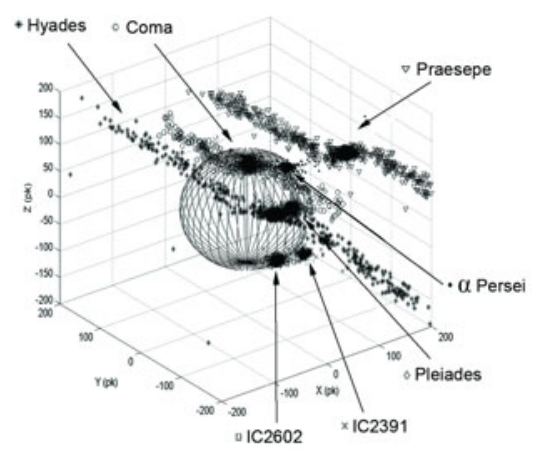

Figure 2. Nearest open star clusters with their tails.

5. Pleiades. The Pleiades cluster lies near the circumsolar sphere (see Fig. 2). However, since the cluster is young, its tail has not yet been completely formed. Our simulations show that the circumsolar sphere may contain several stars.

6. Praesepe, IC 2391, IC 2602, and $\alpha$ Persei clusters. Although Praesepe tail has the length of $\sim 700 \mathrm{pc}$, it is located entirely outside the circumsolar sphere(see Fig. 2). The clusters IC 2391, IC 2602, and $\alpha$ Persei have virtually no tails and lie fairly far from the circumsolar sphere. Mean relative velocities of the tail stars are low $\left(\sim 1 \mathrm{~km} \mathrm{~s}^{-1}\right)$ in the coordinate system associated with the Galactic orbit of the cluster, hence the tail stars may be considered as moving clusters. Thus, dense moving clusters with full space velocity vector close to that of the Hyades and Coma clusters and a sparsely populated cluster with the total velocity vector close to that of the Pleiades can be observed in the circumsolar sphere of radius $100 \mathrm{pc}$.

\section{Acknowledgements}

We thank Dr. O.V. Chumak (Sternberg Astronomical Institute, Moscow State University) and Prof. S. Aarseth (Cambridge University, Great Britain) for the invaluable help and permanent consultations. This work was partly supported by the Russian Foundation for Basic Research (grants 05- 02-16526 and 07-02-00961-) and the Russian Federation President's grant for "Leading Scientific Schools" NSh-389.2003.2.

\section{References}

Aarseth, S. 2003, Gravitational N-Body Simulations (Cambridge Univ. Press, Cambridge)

Chumak, Ya. O., Rastorguev A. S., \& Aarseth, S. 2005, Astron. Lett. 31, 6, 308

Chumak, Ya. O. \& Rastorguev A. S. 2006, Astron. Lett. 32, 3, 157

Chumak, Ya. O. \& Rastorguev, A. S. 2006, Astron. Lett. 32, 7, 446

Nordstrom B., et al. 2004, A \& A 419, 989 